\title{
Bound states and field-polarized Haldane modes in a quantum spin ladder
}

\author{
S. Ward,${ }^{1,2,3}$ P. Bouillot, ${ }^{4,5}$ C. Kollath, ${ }^{3,6}$ T. Giamarchi, ${ }^{3}$ K. P. Schmidt,${ }^{7}$ B. Normand,${ }^{1}$ K. W. \\ Krämer ${ }^{8}$ D. Biner, ${ }^{8}$ R. Bewley, ${ }^{9}$ T. Guidi, ${ }^{9}$ M. Boehm,${ }^{10}$ D. F. McMorrow, ${ }^{2}$ and Ch. Rüegg ${ }^{1,3}$ \\ ${ }^{1}$ Laboratory for Neutron Scattering and Imaging, \\ Paul Scherrer Institut, CH-5232 Villigen PSI, Switzerland \\ ${ }^{2}$ London Centre for Nanotechnology and Department of Physics and Astronomy, \\ University College London, London WC1E 6BT, United Kingdom \\ ${ }^{3}$ Department of Quantum Matter Physics, University of Geneva, CH-1211 Geneva, Switzerland \\ ${ }^{4}$ Department of Medical Imaging and Information Sciences, Interventional Neuroradiology Unit, \\ University Hospitals of Geneva, CH-1211 Geneva, Switzerland \\ ${ }^{5}$ Laboratory for Hydraulic Machines, École Polytechnique Fédérale de Lausanne, CH-1015 Lausanne, Switzerland \\ ${ }^{6}$ HISKP, University of Bonn, Nussallee 14-16, 53115 Bonn, Germany \\ ${ }^{7}$ Theoretische Physik I, Staudtstrasse 7, FAU Erlangen-Nürnberg, 91058 Erlangen, Germany \\ ${ }^{8}$ Department of Chemistry and Biochemistry, University of Bern, CH-3012 Bern, Switzerland \\ ${ }^{9}$ ISIS Facility, Rutherford Appleton Laboratory, Chilton, Didcot, Oxford OX11 0QX, United Kingdom \\ ${ }^{10}$ Institut Laue Langevin, 6 rue Jules Horowitz BP156, 38024 Grenoble CEDEX 9, France
}

(Dated: September 2, 2016)

\begin{abstract}
The challenge of one-dimensional systems is to understand their physics beyond the level of known elementary excitations. By high-resolution neutron spectroscopy in a quantum spin ladder material, we probe the leading multiparticle excitation by characterizing the two-magnon bound state at zero field. By applying high magnetic fields, we create and select the singlet (longitudinal) and triplet (transverse) excitations of the fully spin-polarized ladder, which have not been observed previously and are close analogs of the modes anticipated in a polarized Haldane chain. Theoretical modelling of the dynamical response demonstrates our complete quantitative understanding of these states.
\end{abstract}

PACS numbers: 75.10.Jm, 75.40.Gb, 75.40.Mg, 78.70.Nx

Throughout physics, one-dimensional (1D) systems show a range of intriguing and unconventional phenomena. The dominance of quantum fluctuations in 1D materials makes them the ultimate form of quantum matter. The physics of the ground states and elementary, lowenergy excitations of $1 \mathrm{D}$ systems are by now rather well understood in theory [1] and have been realized in experiment in a number of quite different fields, including conducting wires [2], atomic chains [3], quantum magnets [4], and ultracold atoms [5]. Looking forwards, the next frontier is to understand and control the physics of these systems on all energy scales, including their multiparticle excitations and topological states.

Quantum magnets provide an excellent arena not only for quantitative measurements of the strongly correlated quantum wave function but also for its systematic control by applied external parameters [6-8]. Among the systems whose elementary magnetic excitations are already well characterized, one key model is the $S=1 / 2$ "ladder," consisting of two coupled spin chains [9], with detailed experimental studies performed on genuine ladder materials including $\mathrm{La}_{4} \mathrm{Sr}_{10} \mathrm{Cu}_{24} \mathrm{O}_{41}$ [10], BPCB [1117] and DIMPY [18-21]. The ladder has many parallels to another cornerstone model, the Haldane $(S=1)$ chain [22, 23], and significant progress has been made in calculating their dynamical response [24]. However, genuine Haldane materials with accessible energy scales have proven difficult to find [25-27], and thus the response in strong magnetic fields remains an open problem [28, 29].
In this Letter, we report on measurements of singleand multi-magnon excitations in the spin ladder bispiperidinium copper tetrachloride (BPCC). By exploiting the elegant parity selectivity of the ladder geometry, at zero field we demonstrate the presence of a strong two-magnon bound-state triplet over half of the Brillouin zone and quantify its spectral weight. At high fields, we demonstrate the selection criteria for the singlet excitation, or amplitude mode, of the fully field-polarized (FP) phase, as well as for its triplet (transverse) mode. Both are unknown in a conventional ferromagnet and have direct analogs in the FP Haldane chain. By detailed analytical and numerical modelling, we describe our intensity measurements with quantitative accuracy.

The spin ladder has two basic magnetic interactions, the rung $\left(J_{r}\right)$ and leg $\left(J_{l}\right)$ couplings, and one ratio, $\gamma=J_{l} / J_{r} . \mathrm{BPCB}(\gamma \simeq 0.26)$ and DIMPY $(\gamma \simeq 1.7) \mathrm{ex}-$ emplify contrasting regimes of ladder behavior. The chloride analog of BPCB, $\left(\mathrm{C}_{5} \mathrm{D}_{12} \mathrm{~N}\right)_{2} \mathrm{CuCl}_{4}$ (BPCC, Fig. S2) crystallizes in the monoclinic space group $\mathrm{P} 22_{1} / \mathrm{c}$. Two halide bridges between pairs of $\mathrm{Cu}^{2+}$ ions form the rung dimer $\left(J_{r}\right)$. A further halide bridge between ions repeating periodically in $\hat{a}$ provides $J_{l}$. BPCC is an exceptional realization of a very clean $S=1 / 2$ spin ladder with, as we will show, three attributes ideal for our studies. First, the ladders are well separated by piperidinium groups, making them effectively isolated. Second, the ratio $\gamma \simeq 0.4$ [30] is perfectly suited for observing bound states in the two-magnon sector (whose weight scales with $\gamma^{2}$ ), with- 
out strong interference from the scattering states. Third, the low energy scales in BPCC [30, 31] allow one to work well within the FP phase at laboratory magnetic fields.

High-quality deuterated single-crystal samples were synthesized and five crystals of total mass $2.4 \mathrm{~g}$ were coaligned on the MORPHEUS instrument at SINQ for the collection of inelastic neutron scattering data on the time-of-flight spectrometer LET at ISIS [32]. The sample was mounted inside a $9 \mathrm{~T}$ vertical cryomagnet, on a dilution insert with a stable base temperature of $60 \mathrm{mK}$. Frame-rate multiplication was used with a primary incoming neutron energy of $2.5 \mathrm{meV}$. Data at zero field were collected at 104 sample rotation angles and processed using the MANTID program [33]. The resulting datasets for the dynamical structure factor, $S(\mathbf{Q}, \omega)$, were analyzed with the HORACE software package [34].

In the two-leg ladder, the rung-singlet wave function is antisymmetric whereas the triplets are symmetric (represented schematically in Fig. S1). The geometry therefore allows a full parity selection between odd $\left(q_{y}=\pi\right.$; e.g. singlet-triplet) and even $\left(q_{y}=0\right.$; e.g. triplet-triplet) excitation processes [24, 35], where $q_{y}$ is the wave vector across the ladder unit. There is no mixing between modes of opposite parity and $S(\mathbf{Q}, \omega)$ separates completely into odd- and even-parity sectors, whose intensity maxima occur in very different parts of the Brillouin zone [Figs. 1(d) and 1(e)]. Although the crystallography of BPCC dictates that there are two different ladder orientations, this parity selection remains possible [24, 35], as shown in Sec. S1 of the supplemental material (SM) [36].

We begin our presentation at zero applied field. We refer to the elementary one-triplet excitation of the ladder as a "magnon." The one-magnon dispersion relation is clearly evident in Fig. 1(a), where the four-dimensional (4D) intensity dataset is analyzed to select the $q_{y}=\pi$ sector. Our data treatment is discussed in Sec. S2 of the SM. We observe an excitation with gap $0.2 \mathrm{meV}$, bandwidth $0.23 \mathrm{meV}$, and periodicity $2 \pi$, suggesting that $J_{r}$ is significantly stronger than $J_{l}$.

Several theoretical approaches give good descriptions of the two-leg ladder in the strong-rung regime. From an optimized fit of the one-magnon data to a strongcoupling expansion [37] [Eq. (S5) of Sec. S3], we deduce the exchange parameters $J_{r}=0.295(8) \mathrm{meV}$ and $J_{l}=$ $0.116(1) \mathrm{meV}$; the resulting dispersion is shown as the red lines in Figs. 1(a) and 1(b). The corresponding values from the bond-operator technique [38] [Eq. (S6)] are $J_{r}=0.306(8) \mathrm{meV}$ and $J_{l}=0.113(1) \mathrm{meV}$, and the dispersion is identical within the error bars. Thus the leg exchange in $\mathrm{BPCC}$, with $\gamma \simeq 0.39$, is more significant than in BPCB, which is the key to our quantitative observations of bound states. We perform both DMRG and systematic high-order series-expansion calculations for $S(\mathbf{Q}, \omega)$ (Sec. S3), which capture the contributions of all excitation channels; with optimized parameters $J_{r}=$
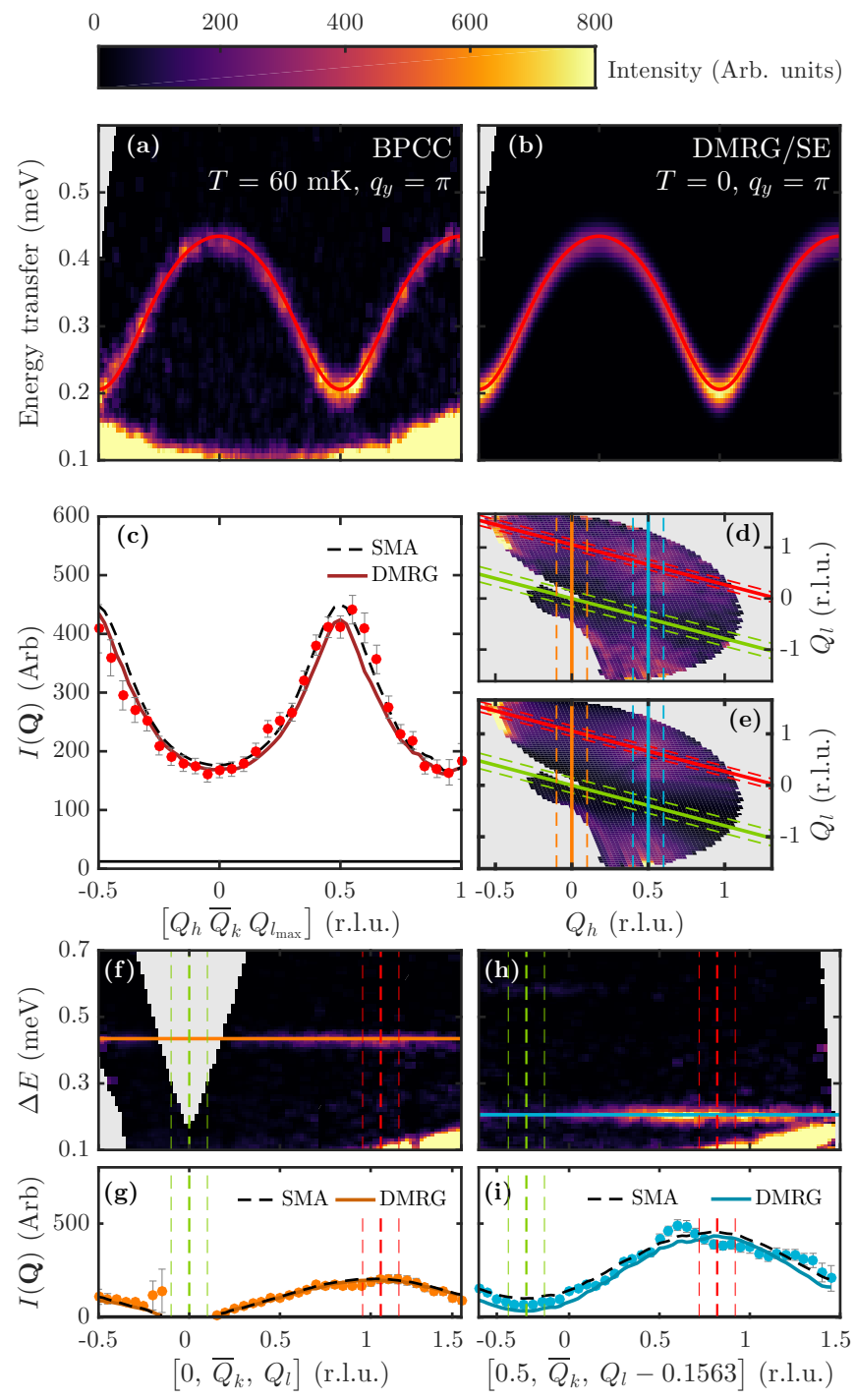

FIG. 1. One-magnon excitations in BPCC. (a) $S(\mathbf{Q}, \omega)$ measured in the $q_{y}=\pi$ sector. (b) $S(\mathbf{Q}, \omega)$ obtained by DMRG and series-expansion (SE) calculations, whose results are indistinguishable. The solid red line is the dispersion obtained from strong-coupling [Eq. (S5)] and bond-operator treatments [Eq. (S6)]. (c) $I(\mathbf{Q})$ obtained by integration over $Q_{k}\left(\right.$ denoted $\left.\bar{Q}_{k}\right)$ and over the energy range $0.19 \leq \omega \leq 0.43$ meV, shown as red points and computed in the SMA (dashed black) and by DMRG (solid red lines); the solid black line denotes the background and $Q_{l_{\max }}$ is defined in Eq. (S4). (d) Measured structure factor and (e) that calculated by DMRG; $\mathbf{Q}$ vectors for the ladder direction in the $q_{y}=\pi$ and $q_{y}=0$ sectors are shown respectively as red and green solid lines, with those transverse to the ladder at $Q_{h}=0$ and 0.5 shown respectively in blue and orange; dashed lines mark the transverse integration ranges. (f) $S(\mathbf{Q}, \omega)$ as a function of $Q_{l}$, taken at $Q_{h}=0$ and cutting the $q_{y}=\pi$ [intensity maxima, red dashed lines, panel (a)] and $q_{y}=0$ [minima, green, Fig. 2(a)] sectors. The solid orange line is the one-magnon band maximum [Eq. (S5)]. (g) $I$ (Q) $(0.40 \leq \omega \leq 0.43 \mathrm{meV}$, orange points), compared with SMA (dashed black) and DMRG calculations (solid orange lines). (h) $S(\mathbf{Q}, \omega)$ measured for scattering vectors $\left[0.5 \bar{Q}_{k} Q_{l}-0.1563\right]$ at the band minimum. (i) $I$ (Q) $(0.19 \leq \omega \leq 0.23 \mathrm{meV}$, blue points), with corresponding theoretical predictions. 
$0.295(0.294) \mathrm{meV}$ and $J_{l}=0.115(0.117) \mathrm{meV}$ for DMRG (series expansions), both yield excellent agreement with the one-magnon measurements, as shown in Fig. 1(b).

For a quantitative discussion of the scattering intensity, we integrate $S(\mathbf{Q}, \omega)$ over specific energy ranges and consider the resulting structure factor, $I(\mathbf{Q})$. The quantity $I(\mathbf{Q})$ obtained by integrating over the full one-magnon energy range $(0.19 \leq \omega \leq 0.43 \mathrm{meV})$ is shown in Fig. $1(\mathrm{c})$. Our DMRG (solid line) and series-expansion calculations reproduce not only the dispersion but also the spectral weight to very high accuracy. To analyze the one-magnon intensity, we exploit the dominance of this mode in the spectrum to apply the single-mode approximation (SMA, Sec. S3). With prior knowledge of $J_{r}$ and $J_{l}$, the average spin correlations, $\left\langle\mathbf{S}_{1} \cdot \mathbf{S}_{2}\right\rangle$, can be extracted separately for the rung and leg bonds [38]. Here, however, the unknown overall scale factor restricts us to deducing their ratio [35], whose expected theoretical value (Sec. S3) is $0.26(1)$. From our data, we obtain a correlation ratio of $0.22(1)$ and the fit to $I(\mathbf{Q})$ shown by the dashed line in Fig. 1(c). Thus our one-magnon intensity measurements both quantify the correlations (entanglement) in the quantum wave function and set the scale factor for intensity calculations in all other scattering sectors.

The intensity distribution for wave vectors transverse to the ladder direction, shown in Figs. 1(f) to 1(i), probes the diagonal $\left(J_{d}\right)$ and interladder $\left(J^{\prime}\right)$ interactions. We find that the one-magnon mode is completely non-dispersive within the instrumental resolution, placing (Sec. S2) an upper bound of $0.006(1) \mathrm{meV}$ on any possible couplings of these types. While this is a maximum of $2 \%$ of $J_{r}$, in fact a far more stringent bound on $J^{\prime}$ is provided by the absence of magnetic order down to $35 \mathrm{mK}$, making the ladders in BPCC at least as isolated as those in BPCB, where $J^{\prime} / J_{r} \approx 0.2 \%$ [17].

Next we consider multi-magnon excitation processes, whose intensity contributions we separate unambiguously by exploiting the parity of the ladder structure (Sec. S1). The symmetric $\left(q_{y}=0\right)$ channel in Fig. 2 is the maximum of all excitation processes changing the triplet count by an even number and is dominated by two-magnon excitations into both continuum states and bound states. The zero-field spectrum of DIMPY has been found to exhibit both features [20]. However, in BPCC the continuum contributions are extremely weak, allowing the dispersion, spectral weight, and termination of the twomagnon bound state to be measured in a degree of detail extremely difficult to achieve either in DIMPY or in the dimerized-chain material $\mathrm{Cu}\left(\mathrm{NO}_{3}\right)_{2} \cdot 2.5 \mathrm{D}_{2} \mathrm{O}$ [42].

$S(\mathbf{Q}, \omega)$ measured with $q_{y}=0$ is shown in Fig. 2(a). We observe a clear, discrete mode lying at an energy of $0.58 \mathrm{meV}$ at $Q_{h}=0.5$, below the continuum edge, and dispersing downwards before disappearing into the continuum, whose contribution is indiscernible in Fig. 2(a). From the selection rules for neutron scattering, this mode is the triplet branch $\left(S_{\text {tot }}=1\right)$ of the two-magnon bound

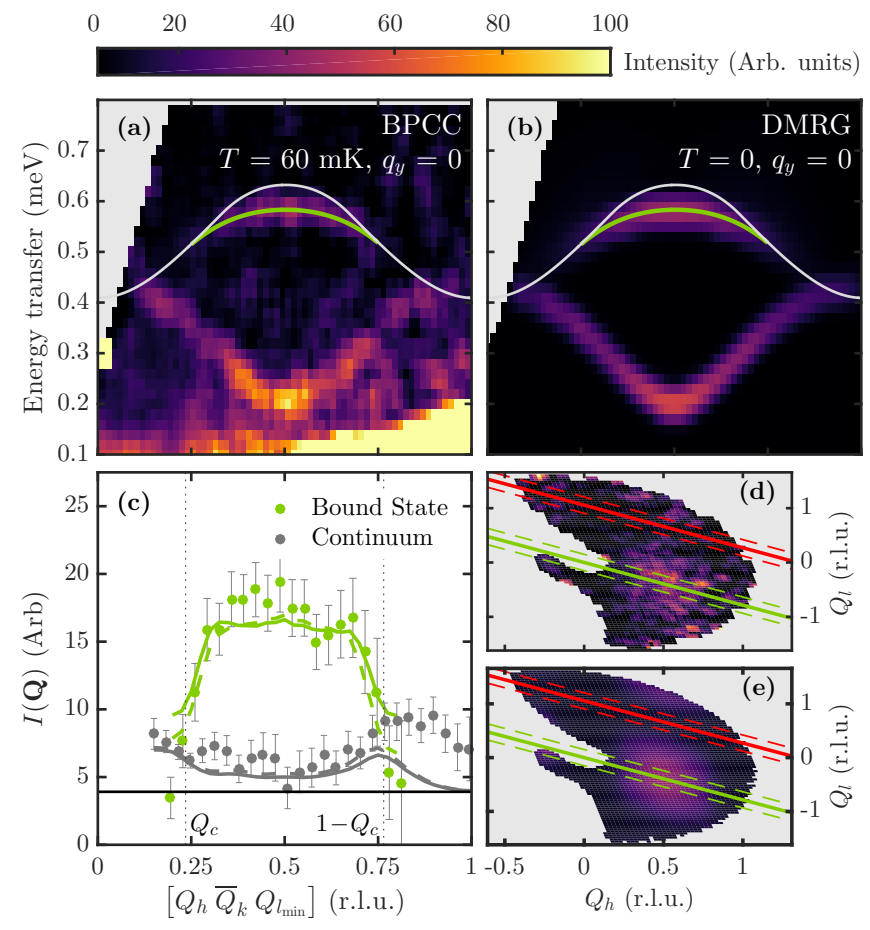

FIG. 2. Two-magnon excitations in BPCC. (a) $S(\mathbf{Q}, \omega)$ measured for $q_{y}=0$. The green line indicates the dispersion [Eq. (S8)] and range [Eq. (1)] over which the triplet component of the two-magnon bound state is expected in BPCC. The white line marks the boundary of the two-magnon continuum [Eq. (S9)]. (b) Corresponding DMRG calculations. (c) Bound-state (green) and continuum (grey) contributions to $I(\mathbf{Q})$, obtained by integrating over energy windows below and above the two-magnon continuum edge; measured intensities (points) are compared with DMRG (solid line) and seriesexpansion (dashed line) calculations. The black line denotes the background and $Q_{l_{\min }}$ is defined in Eq. (S4). Measured (d) and calculated (e) structure factors for the $q_{y}=0$ sector in the $\left(Q_{h}, \bar{Q}_{k}, Q_{l}\right)$ plane; red and green lines as in Fig. 1.

state; the singlet $\left(S_{\text {tot }}=0\right)$ branch is detectable by light scattering [43], and both singlet and quintet $\left(S_{\text {tot }}=2\right)$ branches by neutron spectroscopy at higher temperatures [44]. We note that one-magnon spectral weight is detectable in this sector because the geometry of BPCC excludes perfect destructive interference (Sec. S1). However, in contrast to the discussion of magnon "termination" [39], here the one- and two-magnon excitations are prevented by their opposite parities from mixing where they overlap in energy [as can be seen in Fig. 1(a)].

The bound state overlaps with the two-magnon continuum below a critical wave vector, $Q_{c}$, and decays very readily in this range [40]. It is therefore clearly visible only when $Q_{c}<Q_{h}<1-Q_{c}$, with

$$
Q_{c}=\frac{1}{3}-\frac{5}{4 \sqrt{3} \pi} \gamma-\frac{109}{96 \sqrt{3} \pi} \gamma^{2}+\mathcal{O}\left(\gamma^{3}\right)
$$

in a strong-coupling expansion (Sec. S3). For BPCC, Eq. (1) with $\gamma=0.39$ leads to $Q_{c}=0.212$. However, this 
value of $\gamma$ lies well in the regime where high orders are required for an accurate description. A range of $0.235 \leq$ $Q_{h} \leq 0.765$ is computed in Ref. [40] by working to 12 th order in $\gamma$, and in Fig. 2(b) we require the 10th-order dispersion of the two-magnon bound state [41] and the continuum edge. From our DMRG and series-expansion calculations, we find that both the dispersion [Figs. 2(b) and S3] and the scattered intensity [Figs. 2(c), 2(d), and $2(\mathrm{e})]$ of both the bound-state mode and the two-triplet continuum agree exceptionally well with the data. Thus our results also quantify the termination of the bound state [Fig. 2(c), Sec. S3].

We turn now to the excitation spectrum at high magnetic fields. Quantum magnets with low exchange energies exhibit field-induced critical points marking transitions between several types of exotic quantum phase in materials including $\mathrm{Cs}_{2} \mathrm{CuCl}_{4}$ [45], $\mathrm{CuSO}_{4} \cdot 5 \mathrm{D}_{2} \mathrm{O}$ [46], and BPCB [13, 14, 16]. For two-leg ladders, the physics of the intermediate-field, "Luttinger-liquid" (LL) regime [Fig. 3(c)], which possesses a continuum of gapless, fractional spin excitations, has been studied in depth in $\mathrm{BPCB}$ and we do not repeat this analysis for BPCC here.

However, in all ladder materials studied to date, the dynamical properties have been measured only below (DIMPY, $\left.H_{s} \simeq 29 \mathrm{~T},[20,21]\right)$ or at the saturation field (BPCB, $H_{s}=14 \mathrm{~T}[16]$ ). The exchange parameters of BPCC place full saturation well within our instrumental capabilities. Here we present previously unavailable measurements of the spectrum of the FP ladder.

Figure 3 shows the dynamical structure factor of FP BPCC. Parity remains a good quantum number at high fields and in each sector we find one mode. At $H_{s} \simeq H_{1}$ $=4.067 \mathrm{~T}$, in the $q_{y}=0$ sector we observe [Fig. 3(a)] a mode with a purely sinusoidal dispersion and a gap of $0.3 \mathrm{meV}\left(J_{r}\right)$. On increasing the field to $H_{2}=6.141 \mathrm{~T}$ [Fig. 3(b)], this gap increases linearly with $H$; at $H_{2}$ with $q_{y}=\pi$, we observe a mode with identical dispersion but a gap smaller by precisely $J_{r}$ [Fig. $\left.3(\mathrm{~d})\right]$.

The FP ground state is one of "up-up" triplets, $\left|t_{+}\right\rangle=$ $|\uparrow \uparrow\rangle$, on every ladder rung. Parity selection separates triplet-singlet $\left(q_{y}=\pi\right)$ from triplet-triplet excitations $\left(q_{y}=0\right)$. The excitations of the FP phase are quite unlike the modes of a ferromagnet, with or without an applied field, not least in that the band minimum remains at $q_{h}=\pi$. Although all FP excitations are $\Delta S_{z}=-1$, this can be achieved with $\Delta S_{\text {tot }}=0$ or $\Delta S_{\text {tot }}=-1$. The ladder provides a transparent situation where the latter is simply the singlet excitation of a rung [Fig. S5(a)] and the former the $t_{0}$ triplet [Fig. S5(b)]. The bond-operator method is exact at $H \geq H_{s}$, where quantum fluctuations are completely quenched, giving (Sec. S3)

$$
\begin{aligned}
& \omega_{s}(q)=g \mu_{B}\left(H-H_{s}\right)+J_{l}(1+\cos q), \\
& \omega_{0}(q)=g \mu_{B}\left(H-H_{s}\right)+J_{r}+J_{l}(1+\cos q),
\end{aligned}
$$

with $H_{s}=\left(J_{r}+2 J_{l}\right) / g \mu_{B}$. The upper triplet mode, $t_{-}$, is invisible to neutron scattering and has no dispersion.

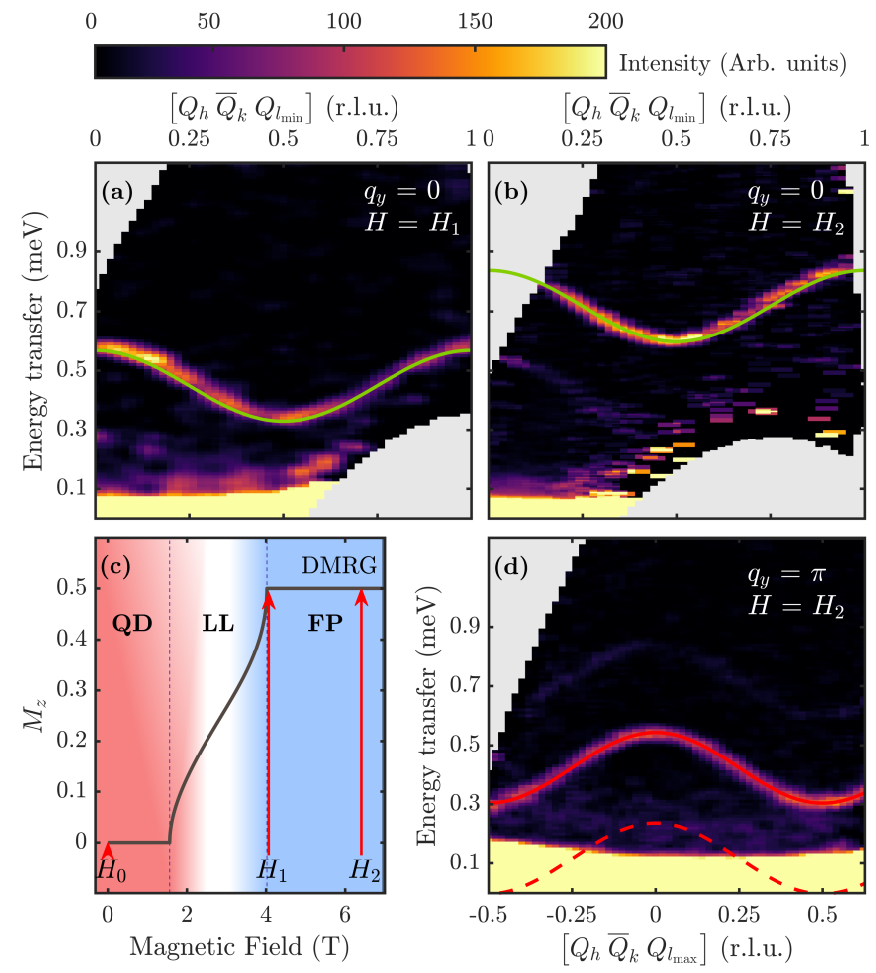

FIG. 3. Excitations in FP BPCC. (a) $S(\mathbf{Q}, \omega)$ measured at $H_{1}=4.067 \mathrm{~T} \simeq H_{s}$ and $(\mathrm{b}) H_{2}=6.141 \mathrm{~T}$, both in the $q_{y}=0$ sector. (c) Magnetization of BPCC (from DMRG) superimposed on the field-induced phase diagram; QD denotes the quantum disordered phase. (d) $S(\mathbf{Q}, \omega)$ measured at $H_{2}$ in the $q_{y}=\pi$ sector. Green and red solid lines are respectively fits to the triplet $\left(q_{y}=0\right)$ and singlet $\left(q_{y}=\pi\right)$ modes using Eq. (2). The dashed line in panel (d) indicates the position of the singlet at $H_{s}$ (not measured), where the spectrum is dominated at low energies by incoherent elastic scattering.

The singlet excitation is a longitudinal, or amplitude, mode of the field-enforced order, and is gapless at $H_{s}$ but has a gap of $H-H_{s}$ above this field; being antisymmetric, it is found with $q_{y}=\pi$, as in Fig. $3(\mathrm{~d})$. This mode is also expected in the Haldane chain, albeit in the adapted form of leg singlets formed from the auxiliary $S=1 / 2$ spins of the AKLT [23] description [Fig. S5(c)]. The $\Delta S=0$ excitation is the gapped triplet, $t_{0}$, a transverse spin mode also exactly analogous to the $t_{0}$ excitation of the FP Haldane chain [Fig. S5(d)]. By its symmetric nature, it is the mode observed in Figs. 3(a) and 3(b), lying higher than the singlet by energy $J_{r}$ [Eq. (2)]. This $t_{0}$ mode has not been observed in any other ladder or Haldane system; it is also expected in FP alternating-chain materials, such as $\mathrm{Cu}\left(\mathrm{NO}_{3}\right)_{2} \cdot 2.5 \mathrm{D}_{2} \mathrm{O}$ [47], although its interpretation is more complicated in the absence of parity selection. By simultaneous fits to Eq. (2) at $H_{1}$ and $H_{2}$, we find that exchange parameters $J_{r}=0.294(1) \mathrm{meV}$ and $J_{l}=0.120(1) \mathrm{meV}$, combined with a $g$ value of $2.26(1)$, provide an extremely accurate account of the data.

We have measured the magnetic excitations of the 
two-leg ladder BPCC at all wave vectors and magnetic fields. At zero field, we exploit the parity-selectivity of the ladder to achieve a total separation of the one- and two-triplet excitation sectors, finding the clearest known example of the two-magnon bound state. In the fully spin-polarized regime, we select the gapped singlet (amplitude) and triplet (phase) modes of the ladder. Thus we provide a systematic understanding of the magnetic excitations in a broad family of gapped 1D quantum magnets, including ladder, alternating-chain, and Haldane systems. For BPCC, we demonstrate that the magnetic response can be modelled with complete quantitative accuracy, in both dispersion and intensity, by modern DMRG and series-expansion techniques.

This work was supported by the Swiss National Science Foundation, the German Research Society (DFG), and the Royal Society. It is based on experiments performed at the Swiss spallation neutron source SINQ at the Paul Scherrer Institute and at the UK spallation neutron source ISIS at the Rutherford-Appleton Laboratory.

[1] T. Giamarchi, Quantum Physics in One Dimension (Oxford University Press, New York, 2004).

[2] O. M. Auslaender, H. Steinberg, A. Yacoby, Y. Tserkovnyak, B. I. Halperin, K. W. Baldwin, L. N. Pfeiffer, and K. W. West, Science 308, 88 (2005).

[3] S. Nadj-Perge, I. K. Drozdov, J. Li, H. Chen, S. Jeon, J. Seo1, A. H. MacDonald, B. A. Bernevig, and A. Yazdani, Science 346, 602 (2014).

[4] H.-J. Mikeska and A. K. Kolezhuk, Lect. Notes Phys. 645, 1 (2004).

[5] M. A. Cazalilla, R. Citro, T. Giamarchi, E. Orignac, and M. Rigol, Rev. Mod. Phys. 83, 1405 (2011); X.-W. Guan, M. T. Batchelor, and C. Lee, Rev. Mod. Phys. 85, 1633 (2013).

[6] see T. Giamarchi, Ch. Rüegg, and O. Tchernyshyov, Nature Physics 4, 198 (2008), and references therein.

[7] S. Ward, P. Bouillot, H. Ryll, K. Kiefer, K. W. Krämer, Ch. Rüegg, C. Kollath, and T. Giamarchi, J. Phys.: Condens. Matter 25014004 (2013).

[8] P. Merchant, B. Normand, K. W. Krämer, M. Boehm, D. F. McMorrow, and Ch. Rüegg, Nature Phys. 10, 373 (2014).

[9] see E. Dagotto and T. M. Rice, Science 271, 618 (1996), and references therein.

[10] S. Notbohm, P. Ribeiro, B. Lake, D. A. Tennant, K. P. Schmidt, G. S. Uhrig, C. Hess, R. Klingeler, G. Behr, B. Büchner, M. Reehuis, R. I. Bewley, C. D. Frost, P. Manuel, and R. S. Eccleston, Phys. Rev. Lett. 98, 027403 (2007).

[11] B. R. Patyal, B. L. Scott, and R. D. Willett, Phys. Rev. B 41, 1657 (1990).

[12] B. C. Watson, V. N. Kotov, M. W. Meisel, D. W. Hall, G. E. Granroth, W. T. Montfrooij, S. E. Nagler, D. A. Jensen, R. Backov, M. A. Petruska, G. E. Fanucci, and D. R. Talham, Phys. Rev. Lett. 86, 5168 (2001).

[13] Ch. Rüegg, K. Kiefer, B. Thielemann, D. F. McMorrow, V. Zapf, B. Normand, M. B. Zvonarev, P. Bouillot,
C. Kollath, T. Giamarchi, S. Capponi, D. Poilblanc, D. Biner, and K. W. Krämer, Phys. Rev. Lett. 101, 247202 (2008).

[14] M. Klanjšek, H. Mayaffre, C. Berthier, M. Horvatić, B. Chiari, O. Piovesana, P. Bouillot, C. Kollath, E. Orignac, R. Citro, and T. Giamarchi, Phys. Rev. Lett. 101, 137207 (2008)

[15] T. Lorenz, O. Heyer, M. Garst, F. Anfuso, A. Rosch, Ch. Rüegg, and K. Krämer, Phys. Rev. Lett. 100, 067208 (2008). F. Anfuso, M. Garst, A. Rosch, O. Heyer, T. Lorenz, Ch. Rüegg, and K. Krämer, Phys. Rev. B 77, 235113 (2008).

[16] B. Thielemann, C. Rüegg, H. M. Rønnow, A. M. Läuchli, J. S. Caux, B. Normand, D. Biner, K. W. Krämer, H. U. Güdel, J. Stahn, K. Habicht, K. Kiefer, M. Boehm, D. F. McMorrow, and J. Mesot, Phys. Rev. Lett. 102, 107204 (2009).

[17] B. Thielemann, Ch. Rüegg, K. Kiefer, H. M. Rønnow, B. Normand, P. Bouillot, C. Kollath, E. Orignac, R. Citro, T. Giamarchi, A. M. Läuchli, D. Biner, K. W. Krämer, F. Wolff-Fabris, V. Zapf, M. Jaime, J. Stahn, N. B. Christensen, B. Grenier, D. F. McMorrow, and J. Mesot, Phys. Rev. B 79, 020408(R) (2009).

[18] A. Shapiro, C. P. Landee, M. M. Turnbull, J. Jornet, M. Deumal, J. J. Novoa, M. A. Robb, and W. Lewis, J. Am. Chem. Soc. 129, 952 (2007).

[19] T. Hong, Y. H. Kim, C. Hotta, Y. Takano, G. Tremelling, M. M. Turnbull, C. P. Landee, H.-J. Kang, N. B. Christensen, K. Lefmann, K. P. Schmidt, G. S. Uhrig, and C. Broholm, Phys. Rev. Lett. 105, 137207 (2010).

[20] D. Schmidiger, P. Bouillot, S. Mühlbauer, S. Gvasaliya, C. Kollath, T. Giamarchi, and A. Zheludev, Phys. Rev. Lett. 108, 167201 (2012); D. Schmidiger, S. Mühlbauer, A. Zheludev, P. Bouillot, T. Giamarchi, C. Kollath, G. Ehlers, and A. M. Tsvelik, Phys. Rev. B 88, 094411 (2013).

[21] D. Schmidiger, P. Bouillot, T. Guidi, R. Bewley, C. Kollath, T. Giamarchi, and A. Zheludev, Phys. Rev. Lett. 111, 107202 (2013).

[22] F. D. M. Haldane, Phys. Rev. Lett. 50, 1153 (1983); Phys. Lett. A 93, 464 (1983).

[23] I. Affleck, T. Kennedy, E. H. Lieb, and H. Tasaki, Phys. Rev. Lett. 59, 799 (1987); Commun. Math. Phys. 115, 477 (1988).

[24] P. Bouillot, C. Kollath, A. M. Läuchli, M. Zvonarev, B. Thielemann, C. Rüegg, E. Orignac, R. Citro, M. Klanjšek, C. Berthier, M. Horvatić, and T. Giamarchi, Phys. Rev. B 83, 054407 (2011).

[25] A. Zheludev, Z. Honda, C. L. Broholm, K. Katsumata, S. M. Shapiro, A. Kolezhuk, S. Park, and Y. Qiu, Phys. Rev. B 68, 134438 (2003).

[26] A. K. Bera, B. Lake, A. T. M. N. Islam, and A. Schneidewind, Phys. Rev. B 92, 060412(R) (2015).

[27] V. S. Zapf, D. Zocco, B. R. Hansen, M. Jaime, N. Harrison, C. D. Batista, M. Kenzelmann, C. Niedermayer, A. Lacerda, and A. Paduan-Filho, Phys. Rev. Lett. 96, 077204 (2006).

[28] A. K. Kolezhuk and H.-J. Mikeska, Prog. Theor. Phys. Suppl. 145, 85 (2002).

[29] F. H. L. Essler and I. Affleck, J. Stat. Mech. P12006 (2004).

[30] H. Ryll, K. Kiefer, C. Rüegg, S. Ward, K. W. Krämer, D. Biner, P. Bouillot, E. Coira, T. Giamarchi, and C. Kollath, Phys. Rev. B 89, 144416 (2014). 
[31] T. Tajiri, H. Deguchi, M. Mito, S. Takagi, H. Nojiri, T. Kawae, and K. Takeda, J. Magn. Magn. Mater. 272-276, 1070 (2004).

[32] R. I. Bewley, J. W. Taylor, and S. M. Bennington, Nuclear Instruments and Methods in Physics Research Section A: Accelerators, Spectrometers, Detectors and Associated Equipment 637, 128 (2011).

[33] Available at http://www.mantidproject.org.

[34] R. A. Ewings, A. Buts, M. D. Le, J. van Duijn, I. Bustinduy, and T. G. Perring, Nuclear Instruments and Methods in Physics Research Section A: Accelerators, Spectrometers, Detectors and Associated Equipment 834, 132 (2016).

[35] B. Thielemann, Ph. D. thesis, ETH Zurich (2009).

[36] See Supplemental Material at [URL will be inserted by publisher] for details.

[37] M. Reigrotzki, H. Tsunetsugu, and T. M. Rice, J. Phys.: Condens. Matter 6, 9235 (1994).

[38] B. Normand and Ch. Rüegg, Phys. Rev. B 83, 054415 (2011), and references therein.

[39] M. B. Stone, I. A. Zaliznyak, T. Hong, C. L. Broholm, and D. H. Reich, Nature 440, 187 (2006).
[40] W. Zheng, C. J. Hamer, R. R. P. Singh, S. Trebst, and H. Monien, Phys. Rev. B 63, 144410 (2001).

[41] C. Knetter, K. P. Schmidt, and G. S. Uhrig, Eur. Phys. J. B 36, 525 (2004).

[42] D. A. Tennant, C. Broholm, D. H. Reich, S. E. Nagler, G. E. Granroth, T. Barnes, K. Damle, G. Xu, Y. Chen, and B. C. Sales, Phys. Rev. B 67, 054414 (2003).

[43] M. Windt, M. Grüninger, T. Nunner, C. Knetter, K. P. Schmidt, G. S. Uhrig, T. Kopp, A. Freimuth, U. Ammerahl, B. Büchner, and A. Revcolevschi, Phys. Rev. Lett. 87, 127002 (2001).

[44] A. Honecker, F. Mila, and B. Normand, to appear in Phys. Rev. B (arxiv:1605.09392).

[45] R. Coldea, D. A. Tennant, K. Habicht, P. Smeibidl, C. Wolters, and Z. Tylczynski, Phys. Rev. Lett. 88, 137203 (2002).

[46] M. Mourigal, M. Enderle, A. Klöpperpieper, J.-S. Caux, A. Stunault, and H. M. Rønnow, Nat Phys 9, 435 (2013).

[47] M. B. Stone, Y. Chen, D. H. Reich, C. Broholm, G. Xu, J. R. D. Copley, and J. C. Cook, Phys. Rev. B 90, 094419 (2014). 


\title{
Supplemental Material for "Bound states and field-polarized Haldane modes in a quantum spin ladder"
}

\author{
S. Ward,${ }^{1,2,3}$ P. Bouillot, ${ }^{4,5}$ C. Kollath, ${ }^{3,6}$ T. Giamarchi, ${ }^{3}$ K. P. Schmidt,${ }^{7}$ B. Normand, ${ }^{1}$ K. W. \\ Krämer, ${ }^{8}$ D. Biner, ${ }^{8}$ R. Bewley, ${ }^{9}$ T. Guidi, ${ }^{9}$ M. Boehm,${ }^{10}$ D. F. McMorrow,${ }^{2}$ and Ch. Rüegg ${ }^{1,3}$ \\ ${ }^{1}$ Laboratory for Neutron Scattering and Imaging, \\ Paul Scherrer Institut, CH-5232 Villigen PSI, Switzerland \\ ${ }^{2}$ London Centre for Nanotechnology and Department of Physics and Astronomy, \\ University College London, London WC1E 6BT, England, United Kingdom \\ ${ }^{3}$ Department of Quantum Matter Physics, University of Geneva, CH-1211 Geneva, Switzerland \\ ${ }^{4}$ Department of Medical Imaging and Information Sciences, Interventional Neuroradiology Unit, \\ University Hospitals of Geneva, CH-1211 Geneva, Switzerland \\ ${ }^{5}$ Laboratory for Hydraulic Machines, École Polytechnique Fédérale de Lausanne, CH-1015 Lausanne, Switzerland \\ ${ }^{6}$ HISKP, University of Bonn, Nussallee 14-16, 53115 Bonn, Germany \\ ${ }^{7}$ Theoretische Physik I, Staudtstrasse 7, FAU Erlangen-Nürnberg, 91058 Erlangen, Germany \\ ${ }^{8}$ Department of Chemistry and Biochemistry, University of Bern, CH-3012 Bern, Switzerland \\ ${ }^{9}$ ISIS Facility, Rutherford Appleton Laboratory, Chilton, Didcot, Oxford OX11 0QX, United Kingdom \\ ${ }^{10}$ Institut Laue Langevin, 6 rue Jules Horowitz BP156, 38024 Grenoble CEDEX 9, France \\ PACS numbers: $75.10 . \mathrm{Jm}, 75.40 . \mathrm{Gb}, 75.40 . \mathrm{Mg}, 78.70 . \mathrm{Nx}$
}

\section{S1. Neutron Scattering in a Two-Leg Spin Ladder \\ Parity Selection}

A fundamental consequence of the two-leg geometry is that all excitations of the spin ladder have an exact even or odd parity. Excitations between singlet and triplet states have odd parity $\left(q_{y}=\pi\right.$ sector $)$ and may therefore be separated systematically from excitations of intertriplet (or inter-singlet) character, which have even parity $\left(q_{y}=0\right.$ sector $)$; this situation is represented schematically in Fig. S1. Most important for the present purposes is that one-magnon excitations are odd whereas two-magnon excitations are even; these opposite parities result in opposite phases for constructive or destructive interference, and hence in a complete separation of the maximal intensities of the two sectors in reciprocal space. A further consequence is that excitations of opposite parities do not mix, excluding the possibility of "quasiparticle breakdown" where they overlap.

\section{Crystal Structure of BPCC}

The structure of BPCC has the monoclinic space group $\mathrm{P} 22_{1} / \mathrm{c}$, with lattice parameters determined by neutron diffraction at $1.6 \mathrm{~K}$ as $a=8.08657(5) \AA, b=16.57703(9)$ $\AA, c=12.14457(7) \AA$, and $\beta=102.2395(4)^{\circ}$ [S1]. The unit cell contains four $\mathrm{Cu}^{2+}$ atoms $(S=1 / 2)$, which form two spin dimers constituting the ladder rungs, as shown in Fig. S2(a). These rung dimer units repeat periodically along the $\hat{a}$ direction, with an exchange interaction that forms the ladder legs [Fig. S2(a)]. Ladders neighboring in the $\hat{b}$ direction are related by a $2{ }_{1}$ screw axis and hence there are two types of spin ladder, identical (by symmetry) in their exchange interactions but different in orientation [Fig. S2(b)]. The inequivalent rung vectors are given by

$$
\mathbf{r}_{1,2}=[0.3822 \pm 0.1730 .4866] ;
$$

in the same units, the leg vector is $\left[\begin{array}{lll}1 & 0 & 0\end{array}\right]$. Despite this inequivalence, parity remains a good quantum number: excitations in the 0 and $\pi$ sectors are associated with geometrical phase factors expressing their constructive or destructive interference, which determine the precise locations of maximal and minimal scattering intensity throughout the Brillouin zone for each sector. Because of the monoclinic structure of BPCC, these maxima and minima are determined not solely by the reciprocal-space component, $Q_{h}$, for the ladder direction, but are found respectively at $\left[\begin{array}{lll}Q_{h} & 0 & Q_{l_{\max }}\end{array}\right]$ and $\left[\begin{array}{lll}Q_{h} & 0 & Q_{l_{\min }}\end{array}\right]$, where $Q_{l_{\max }}$ and $Q_{l_{\min }}$ depend on $Q_{h}$, as we discuss below.

\section{Scattering Cross-Section}

The complete separation of excitations into even (0) and odd $(\pi)$ parity sectors means that the neutron scattering cross section may also be decomposed into two separate types of contribution, $S_{0}^{\alpha \beta}$ and $S_{\pi}^{\alpha \beta}$ [S3]. Although $\alpha$ and $\beta$ denote general spin indices, for pure Heisenberg interactions one has $S^{x x}=S^{y y}=S^{z z}=S^{+-}=S^{-+}$. When the two inequivalent wave vectors for the ladder rungs are taken into account, the total neutron scattering cross-section can be written as

$$
\begin{aligned}
\frac{\mathrm{d}^{2} \sigma}{\mathrm{d} \Omega \mathrm{d} E^{\prime}} \propto & 4\left(1-\frac{Q_{z}^{2}}{Q^{2}}\right)\left[c(\mathbf{Q}) S_{0}^{z z}+s(\mathbf{Q}) S_{\pi}^{z z}\right] \\
& +\left(1+\frac{Q_{z}^{2}}{Q^{2}}\right)\left[c(\mathbf{Q})\left(S_{0}^{+}+S_{0}^{+}\right)+s(\mathbf{Q})\left(S_{\pi}^{+}+S_{\pi}^{++}\right)\right]
\end{aligned}
$$




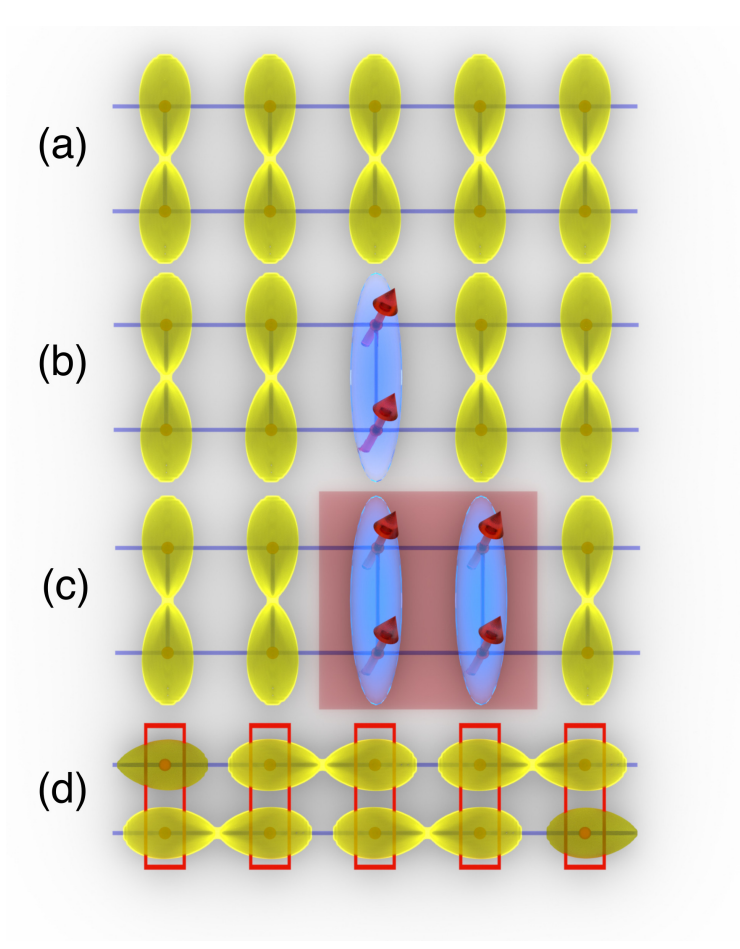

FIG. S1. Schematic representation of rung states and their symmetries in spin-ladder and Haldane-chain systems at zero field. (a) Ground state of antisymmetric rung singlets in the two-leg ladder. (b) One-magnon (symmetric, triplet) excited state. (c) Two-magnon excited state; the triplet pair may be in a net singlet, triplet, or quintet $\left(S_{\text {tot }}=0,1\right.$, or 2$)$ state but its parity is equal to that of the ground state and opposite to that of the one-magnon excitation. (d) Ground state of the Haldane chain in the AKLT representation [S2] of auxiliary $S=1 / 2$ spin pairs at each site; the red boxes represent the operator projecting these spins to a real $S=1$ state.

where $s(\mathbf{Q})=\sum_{i=1,2} \sin ^{2}\left(\mathbf{Q} \cdot \mathbf{r}_{i} / 2\right)$ and $c(\mathbf{Q})=$ $\sum_{i=1,2} \cos ^{2}\left(\mathbf{Q} \cdot \mathbf{r}_{i} / 2\right)$ are phase factors and $Q_{z}$ is the outof-plane wave-vector component. In practice, we focus directly on the dynamical structure factor, $S(\mathbf{Q}, \omega)$, extracted from $\mathrm{d}^{2} \sigma / \mathrm{d} \Omega \mathrm{d} E^{\prime}$.

Equation (S2) may be used to quantify the statements made in the preceding subsections. The maximum in the structure factor contributed by one-magnon excitations is found by selecting the wave vectors $\mathbf{Q}$ maximizing $1-$ $\cos (\mathbf{Q} \cdot \mathbf{r})$, where $\mathbf{r}=\mathbf{r}_{1}-\mathbf{r}_{2}$ is the difference between the inequivalent rung vectors, i.e. $\mathbf{Q}$ must satisfy

$$
2 \pi(\mathbf{Q} \cdot \mathbf{r})=(2 n+1) \pi,
$$

where $n$ is an integer. Conversely, the minimum in the one-magnon structure factor, which coincides with the maximum in the two-magnon (even-parity) channel, is found when $n$ takes a half-integer value. One may then deduce that

$$
Q_{l}=\left[n+1 / 2-0.3822 Q_{h}\right] / 0.4866,
$$

with $n$ an integer for $Q_{l_{\max }}$ and $n$ half-integral for $Q_{l_{\min }}$. (a)

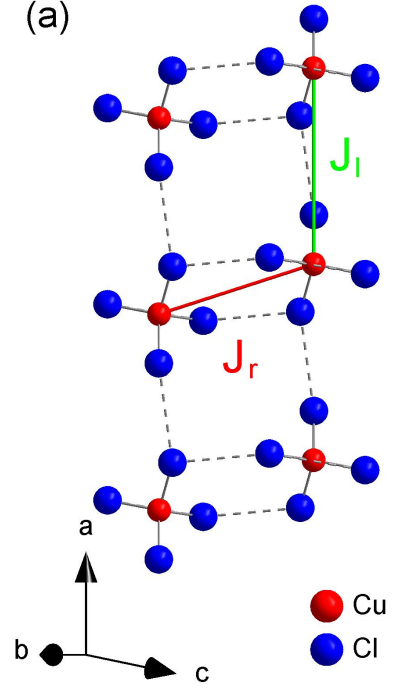

(b)

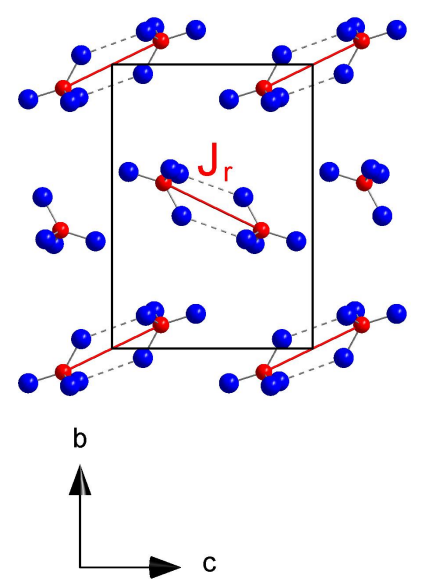

FIG. S2. Representation of the crystal structure of BPCC, highlighting (a) the positions of $\mathrm{Cu}$ and $\mathrm{Cl}$ atoms in a single ladder unit and (b) the relative positions of the different ladder units, which have two different orientations; the piperidinium cations between the ladders are omitted for clarity.

This information is represented in Fig. S3, which shows the simulated structure factor of the one-magnon excitation, i.e. the quantity $S(\mathbf{Q}, \omega(\mathbf{Q}))$. The calculation of the dispersion relation, $\omega(\mathbf{Q})$, and of the corresponding intensity is deferred to Sec. S3. The red and green solid lines mark respectively the lines of maxima and minima of the one-magnon excitations of the spin ladder, whose dynamical structure factor $[S(\mathbf{Q}, \omega)]$ is shown in Fig. 1(a) of the main text. As noted above, the one-magnon minimum is the two-magnon maximum, for which $S(\mathbf{Q}, \omega)$ is shown in Fig. 2(a). Blue solid lines mark the maxima of the one-magnon excitations transverse to the ladder $\left(Q_{l}\right)$, which are obtained at the zone boundary in $Q_{h}$ and the spin-gap energy in $\omega$, and are studied in Fig. 1(h); orange lines mark the minima, which are found at the zone center and the band maximum [Fig. 1(f)].

\section{S2. Neutron Scattering Intensity Analysis}

Experiments were performed on the time-of-flight spectrometer LET, with the BPCC sample mounted in the (horizontal) $a c$ scattering plane. Data at zero applied magnetic field were collected for 104 rotation angles, corrected for detector efficiency and scattered-to-incident wave-vector ratio, $k_{f} / k_{i}$, using the MANTID program [S4], and combined into a "four-dimensional" (4D, meaning three spatial dimensions and one energy) dataset using the Matlab-based HORACE analysis code [S5]. The extent of the resulting dataset in reciprocal-space dimensions $Q_{h}$ and $Q_{l}$ is represented by the white line in 


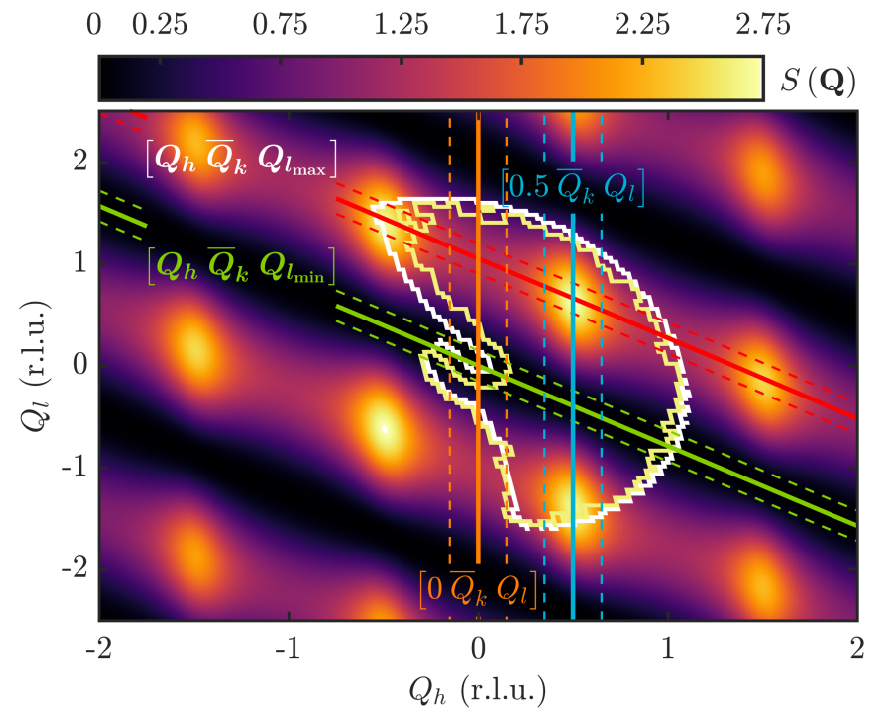

FIG. S3. False-color representation of the simulated structure factor, $S(\mathbf{Q}, \omega)$, of the one-magnon excitation in BPCC, calculated in a strong-coupling analysis and using the singlemode approximation (Sec. S3). The scattered intensity is shown in the horizontal scattering plane, $\left(Q_{h}, 0, Q_{l}\right)$, for the energy value $\omega=\omega(\mathbf{Q})$ of the one-magnon dispersion and for $Q_{k}=0$. The white line marks the full extent of the available data in $Q_{h}$ and $Q_{l}$, which is determined by the detector coverage at the energy of the one-magnon mode. At the (higher) energy of the two-magnon continuum, the detector coverage has shrunk and is shown in yellow. The other lines are described in the text.

Fig. S3. For all of the results presented in the main text, the data were integrated over the $Q_{k}$ direction, representing out-of-plane scattering, where all modes are entirely non-dispersive; we refer to the results of this integration as a 3D dataset and denote this treatment of the $Q_{k}$ direction by $\bar{Q}_{k}$. Further integration over directions along or across the ladder was performed to obtain the final datasets; the dashed lines in Fig. S3 mark these transverse integration ranges. In all cases, the integration range was \pm 0.125 r.l.u., a value achieving an acceptable signal-to-noise ratio while preserving mode coherence and sector separation.

For all quantitative analyses of the scattered intensity, we integrate $S(\mathbf{Q}, \omega)$ over a chosen window of energy to obtain the structure factor, $I(\mathbf{Q})$. In addition to improving very significantly the signal-to-noise ratio, this procedure also allows us to select the energy range in such a way as to remove as much as possible of the contamination due to elastic and multiple scattering events. This last includes scattering from the cryomagnet, which is responsible for the parabolic intensity visible at low energies in Figs. 1(a) and 2(a). The remaining background, due to detector noise and incoherent phonon scattering, is marked by the solid black lines in Figs. 1(c) and 2(c) of the main text. Fits to the models and numerical calculations were optimized by a least-squares approach.

\section{Diagonal and Interladder Interactions}

Exchange interactions between sites in neighboring spin ladders are reflected in the dispersion of the onemagnon mode for $\mathbf{Q}$ vectors transverse to the ladder axis. Any asymmetric exchange interactions within the ladder plaquettes, to which we refer here as "diagonal," would also be manifest as a periodic modulation of the intensity distribution for the same $\mathbf{Q}$ vectors. Figure $1(\mathrm{f})$ shows $S(\mathbf{Q}, \omega)$ along $\left[\begin{array}{lll}0 & 0 & Q_{l}\end{array}\right]$, where the mode is clearly non-dispersive to within the resolution of our measurements. The red line is a theoretical result (Sec. S3) with an interladder coupling of zero, and in fact the fitting process places an upper bound on interladder exchange of $0.006(1) \mathrm{meV}$. The corresponding structure factor, Fig. $1(\mathrm{~g})$, is very well reproduced by calculations performed in the SMA and with DMRG. For transverse wave vectors chosen at the one-magnon gap, the dispersion is again completely flat [Fig. 1(h)] and the structure factor is described perfectly by a system of pure and isolated ladders [Fig. 1(i)]. Again there is no discernible periodicity, in dispersion or intensity, within the instrumental resolution, placing the same limit, $0.006(1) \mathrm{meV}$, on any possible diagonal or interladder interactions.

\section{S3. Theoretical Analysis of Strong-Rung Ladders}

\section{One-Magnon Dispersion}

Several theoretical approaches are known to give good descriptions of the two-leg ladder in the strong-rung regime, including a direct perturbative expansion [S6] and the bond-operator formalism [S7]. Numerically, both exact diagonalization (ED) and DMRG work well because of the short correlation length of the well-gapped system [S8]. In terms of the parameter $\gamma=J_{l} / J_{r}$, to third order in a perturbative expansion one obtains the dispersion relation [S6]

$$
\begin{aligned}
\frac{\omega(q)}{J_{r}}= & 1+\frac{3}{4} \gamma^{2}+\frac{3}{8} \gamma^{3}+\left(\gamma-\frac{1}{4} \gamma^{3}\right) \cos q \\
& -\frac{1}{4}\left(\gamma^{2}+\gamma^{3}\right) \cos 2 q+\frac{1}{8} \gamma^{3} \cos 3 q
\end{aligned}
$$

for the elementary triplet (one-magnon) excitation, where we have defined $q=2 \pi Q_{h}$. In the bond-operator description, the dispersion is given by

$$
\omega(q)=\sqrt{\left(\frac{1}{4} J_{r}-\mu\right)\left(\frac{1}{4} J_{r}-\mu+2 J_{l} \bar{s}^{2} \cos q\right)},
$$

where the $\mu$ is the chemical potential for the triplet excitations, which are hard-core bosons, and $\bar{s}$ expresses the extent to which the ground state is one of pure rung singlets [S7]. 


\section{Single-Mode Approximation}

While Eq. (S2) is an expression for the total neutron scattering cross-section, in many gapped quantum magnets the overwhelming majority of the scattered intensity can be found in the one-magnon branch of the excitation spectrum. In this situation, a meaningful analysis of the spectral weight is obtained within the single-mode approximation (SMA) [S9]. Quite generally, the firstmoment sum rule relates the integrated spectral weight of a spin ladder to the equal-time correlation functions in the form

$$
\begin{gathered}
\int d \omega \hbar \omega S(\mathbf{Q}, \omega)=-\frac{2}{3}\left(J_{r}\left\langle\mathbf{S}_{i}^{m} \cdot \mathbf{S}_{i}^{n}\right\rangle[1-\cos (\mathbf{Q} \cdot \mathbf{d})]\right. \\
\left.+J_{l}\left(\left\langle\mathbf{S}_{i}^{m} \cdot \mathbf{S}_{i+1}^{m}\right\rangle+\left\langle\mathbf{S}_{i}^{n} \cdot \mathbf{S}_{i+1}^{n}\right\rangle\right)\left[1-\cos \left(\mathbf{Q} \cdot \mathbf{r}_{l}\right)\right]\right)
\end{gathered}
$$

where $m$ and $n$ denote the two chains of the ladder, $\mathbf{d}=\mathbf{r}_{m}-\mathbf{r}_{n}$ is the dimer (rung) bond vector and $\mathbf{r}_{l}=\mathbf{r}_{m, i+1}-\mathbf{r}_{m, i}$ is the leg bond vector. In the SMA, the integral over $\omega$ includes a $\delta$-function, $\delta(\omega-\omega(\mathbf{Q}))$, which transforms the left-hand side into a simple product of $\omega(\mathbf{Q})$ and the structure factor, $I(\mathbf{Q})$. The right-hand side is a set of "bond energies," weighted by cosine factors determined from the geometry of the system. These bond energies are the product of the exchange interactions for each bond with the average bond spin correlations.

As noted in the main text, $J_{r}$ and $J_{l}$ for BPCC can be established independently by using the one-magnon dispersion, and therefore one would wish to determine the average spin correlations on the rung and leg bonds directly from the experimental dataset. For guidance, by performing ED calculations on ladders of coupling ratio $\gamma=0.39$ and up to $2 \times 12$ spins using the ALPS package [S10], we determine the average rung spin correlations, $\left\langle\mathbf{S}_{i}^{m} \cdot \mathbf{S}_{i}^{n}\right\rangle=-0.6740$, and leg correlations, $\left\langle\mathbf{S}_{i}^{m} \cdot \mathbf{S}_{i+1}^{m}\right\rangle=\left\langle\mathbf{S}_{i}^{n} \cdot \mathbf{S}_{i+1}^{n}\right\rangle=-0.1816$. However, because of an unknown intensity scale factor, only the ratio of these correlations can be extracted from the measured integrated intensities. From the reduced dataset, we deduce the ratio $\left\langle\mathbf{S}_{i}^{m} \cdot \mathbf{S}_{i+1}^{m}\right\rangle /\left\langle\mathbf{S}_{i}^{m} \cdot \mathbf{S}_{i}^{n}\right\rangle=0.22(1)$, which compares favorably with the ratios 0.2586 , given within the bond-operator formalism [S7], and 0.2694 obtained from ED. The structure factors obtained in the SMA by using these average bond correlation values are entirely consistent with the measured intensities, as shown by the black, dashed lines in Figs. 1(c), 1(g), and 1(i).

\section{Density-Matrix Renormalization Group}

DMRG calculations of the dynamical structure factor of an ideal spin ladder were performed in both the $q_{y}=0$ and $\pi$ sectors using the optimized exchange interaction parameters $J_{r}=0.295 \mathrm{meV}$ and $J_{l}=0.115 \mathrm{meV}$. The calculations used the time-dependent DMRG method [S11S14] to determine the spatial spin correlation functions at zero temperature in real-time for a ladder of 200 rungs. They employed a fixed maximal number of a few hundred states and a maximal simulation time of $200 \hbar / J_{r}$, with a time-step typically taken as $\delta t=0.2 \hbar / J_{r}$. These choices ensured that the remaining errors were sufficiently small as to have no discernible effect on the primary spectral structures. The dynamical spin correlations in momentum space were obtained by Fourier transformation of the spin correlation functions. More details of the calculational procedure are described in Refs. [S15, S16].

The spectra obtained in this way were convolved with a Gaussian function representing the instrumental resolution, combined using Eq. (S2), weighted by the magnetic form factor of $\mathrm{Cu}^{2+}$, and added to a constant background value extracted from the experimental dataset. By this process we formed a DMRG dataset for $S(\mathbf{Q}, \omega)$ completely equivalent to the experimental one. This DMRG dataset was cut, integrated in $\mathbf{Q}$, and integrated in $\omega$ using precisely the same techniques as described above. The results are displayed both as the color contours in Figs. 1(b) and 2(b) of the main text [for $S(\mathbf{Q}, \omega)]$ and as the solid lines in Figs. 1(c), 1(g), 1(i), and 2(c) [for $I(\mathbf{Q})$ ].

\section{High-Order Series Expansions}

The systematic extension of the strong-coupling approximation is the high-order series-expansion method, which has been applied previously for the calculation of bound states and dynamical correlation functions in quantum spin ladders [S17, S18]. Here we have used perturbative continuous unitary transformations (pCUTs) [S18, S19] to map the spin ladder order by order in $\gamma$ to an effective Hamiltonian, $\mathcal{H}_{\text {eff }}$, which conserves the number of elementary $S=1$ excitations. Physically, the $\gamma=0$ ground state is the product state of rung singlets and the elementary excitation is a rung triplet; at finite $\gamma$, these triplets become dressed by their mutual interactions to yield the elementary magnon excitations.

Because $\mathcal{H}_{\text {eff }}$ conserves the magnon number, each (interacting) few-magnon problem can be addressed separately. Here we have determined the one-magnon hopping amplitudes up to order 11 in $\gamma$, the two-magnon interaction amplitudes with $S_{\text {tot }}=1$ up to order 10 , and the matrix elements in the one- and two-magnon channels relevant for $S(\mathbf{Q}, \omega)$ also to 10th order. All series are essentially converged for $\gamma=0.39$ and no extrapolations are required. The one-magnon sector is readily diagonalized by Fourier transformation to give the one-magnon dispersion and spectral weight directly.

In the two-magnon channel, it is necessary to solve a two-body problem to obtain $S(\mathbf{Q}, \omega)$ [S20], which we execute by Lanczos tridiagonalization of the two-magnon $\mathcal{H}_{\text {eff }}$ for fixed total momentum $\mathbf{Q}$. The only remaining degree of freedom is the separation, $d$, of the two magnons, and by allowing a maximal $d=4000$ all finite- 


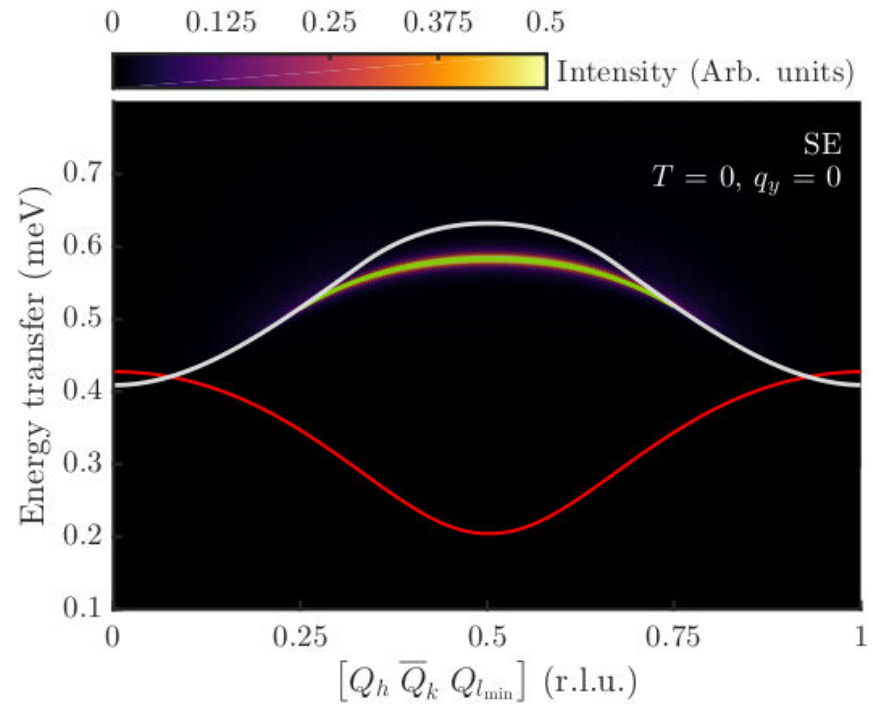

FIG. S4. Dynamical structure factor, $S(\mathbf{Q}, \omega)$, in the symmetric $\left(q_{y}=0\right)$ sector, calculated by series-expansion (SE) methods up to 10th order, showing the triplet component of the two-magnon bound state and the absence of visible intensity from the two-magnon continuum. The red line marks the one-magnon dispersion and the white line the lower boundary of the two-magnon continuum.

size effects are essentially eliminated from the calculation. From the Lanczos tridiagonalization, we extract [S20] the complete two-magnon contribution to $S(\mathbf{Q}, \omega)$, the dispersion and the intensity of the two-magnon bound state as a function of $\mathbf{Q}$, and the corresponding intensity of the two-magnon continuum. We note that the two-magnon structure factor, $I(\mathbf{Q})$, corresponds to the sum of boundstate and continuum contributions and is obtained directly from the matrix elements.

As for our DMRG calculations, to obtain a dataset comparable with experiment we scale and smooth our series-expansion data for $S(\mathbf{Q}, \omega)$ and integrate them in the same manner. Focusing on the results for the twomagnon triplet bound state, in Fig. S4 we show $S(\mathbf{Q}, \omega)$ calculated in the $q_{y}=0$ sector and in Fig. 2(c) of the main text the corresponding $I(\mathbf{Q})$ for comparison with the results from experimental measurements and DMRG calculations.

\section{Two-Magnon Bound States}

For an accurate description of the two-magnon bound state we employ the strong-coupling series expansion [S17, S18]. For illustration, the energy of the triplet component of the bound state is given to third order by

$$
\begin{aligned}
\frac{\omega_{B}(q)}{J_{r}}= & 2-\frac{3}{2} \gamma+\frac{11}{8} \gamma^{2}+\frac{17}{16} \gamma^{3}-\left(\gamma+\frac{1}{4} \gamma^{2}-\frac{9}{16} \gamma^{3}\right) \cos q \\
& -\frac{1}{2}\left(\gamma^{2}+\gamma^{3}\right) \cos 2 q-\frac{5}{16} \gamma^{3} \cos 3 q .
\end{aligned}
$$

In the same approximation, the lower edge of the twomagnon continuum is located at the energy

$$
\begin{aligned}
\frac{\omega_{2 m}(q)}{J_{r}}= & 2+\frac{3}{2} \gamma^{2}+\frac{3}{4} \gamma^{3}-2\left(\gamma-\frac{1}{4} \gamma^{3}\right) \cos \frac{1}{2} q \\
& -\frac{1}{2}\left(\gamma^{2}+\gamma^{3}\right) \cos q-\frac{1}{4} \gamma^{3} \cos \frac{3}{2} q .
\end{aligned}
$$

One observes that the triplet bound states are cut off by the presence of the continuum, but exist over a range of wave vectors $q_{c} \leq q \leq 2 \pi-q_{c}$ around the edges of the Brillouin zone, where $q_{c}=2 \pi Q_{c}$ is given in Eq. (1) of the main text. For ladders with stronger rung-to-leg coupling ratio (weaker $\gamma$ ), $q_{c} \simeq 2 \pi / 3$ and the bound state is restricted to the outer $1 / 3$ of the $1 \mathrm{D}$ Brillouin zone. For larger $\gamma$ values, the range over which the bound-state mode exists (1) becomes slightly larger [S17] and so does its separation in energy from the continuum edge. However, the primary factor affecting its visibility is the intensity of the two-magnon continuum, which rises significantly more strongly than the energetic separation. The very weak two-magnon continuum [Figs. S4 and 2(c)] is therefore the reason why the bound state is so much more clearly visible in BPCC than in any other systems studied previously.

Quantitatively, as discussed in the main text we find that, even for a coupling ratio as apparently modest as $\gamma \simeq 0.4$, a 10th-order expansion is required for an accurate account of the relative positions of the bound state and the continuum edge for a pair of magnons. In our analysis of the scattering intensities in Fig. 2(c), we compute the structure factor by integrating over an energy window up to the two-magnon continuum boundary and attribute these contributions to the bound state. The energy window above the continuum edge is attributed to two-magnon scattering states. It is clear that applying the same process to our $S(\mathbf{Q}, \omega)$ data, calculated both by DMRG and by series expansions, gives an excellent quantitative account of the measured intensities.

We remark here that one of the open questions under these circumstances concerns the "termination" of the bound-state mode where it meets the continuum at the cut-off wave vector, $q_{c}$. As noted in the main text, the termination of the one-magnon mode in the two-magnon continuum, as discussed in Ref. [S21], is precluded in BPCC by their opposite parities. However, both bound and scattering states of two magnons appear in the same parity sector $\left(q_{y}=0\right)$, and thus there is no parity protection for the bound state in this situation. To understand whether the bound state ceases to exist as a welldefined excitation of the system at $q_{c}$, before it, or persists in some form into the two-magnon continuum, it is necessary to analyze the line width of this spectral feature; a structure-factor analysis of the type performed in Fig. 2(c) does not address the origin of the intensity contributions. Unfortunately, the data quality of our experimental measurements is not sufficient for a quantitative 


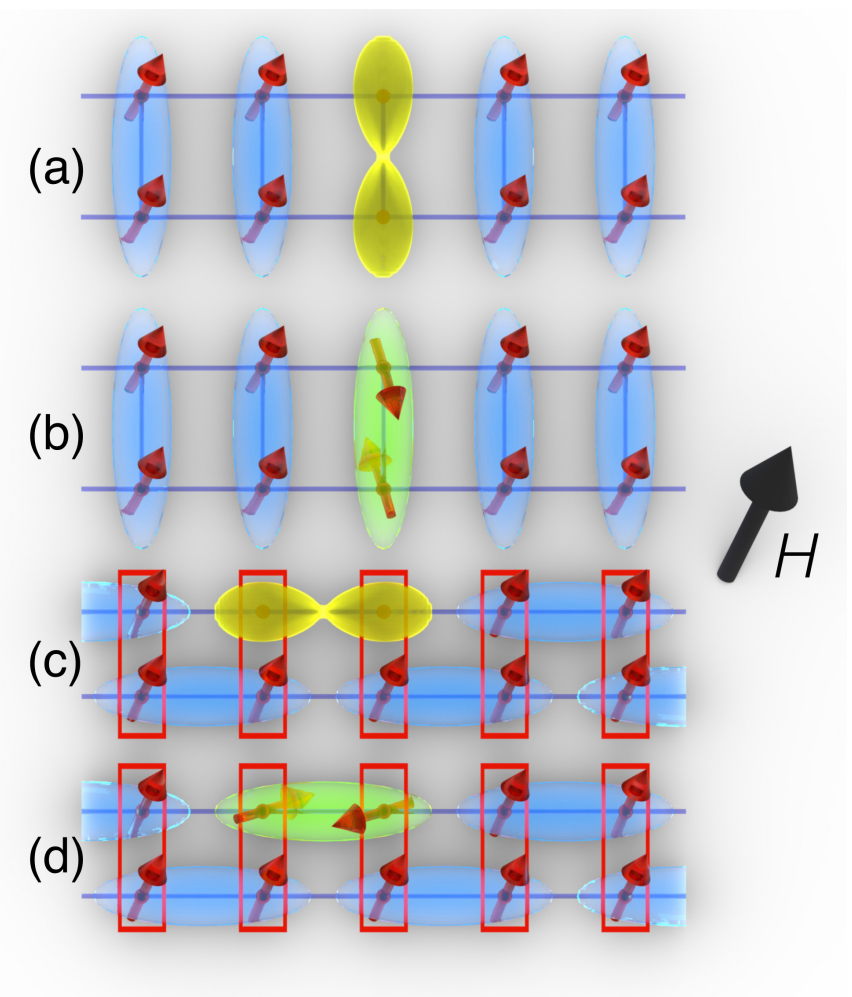

FIG. S5. Schematic representation of the rung states and their symmetries in fully field-polarized spin-ladder and Haldane-chain systems. (a) Polarized two-leg ladder with one rung-singlet excitation. (b) Polarized ladder with one rungtriplet $\left(t_{0}\right)$ excitation. Polarized Haldane chain in the AKLT representation containing (c) one bond-singlet excitation and (d) one $t_{0}$ bond-triplet excitation.

discussion of this issue. Numerically, both our DMRG and series-expansion calculations may be used to investigate the nature of this termination, which, however, lies beyond the scope of the present analysis.

\section{Field-Polarized Phase}

The most straightforward description of the fully polarized (FP) phase is obtained by using the bondoperator description. For magnetic fields beyond the saturation field, $h_{s}=g \mu_{\mathrm{B}} H_{s}$, there are one singlet and two triplet excitations, while the third triplet, $t_{+}$, is the ground state and therefore is treated as fully condensed, i.e. $\left\langle t_{+}\right\rangle=\bar{t}$ is a constant. From the Hamiltonian,

$$
\begin{aligned}
H= & \sum_{i}\left[\left(-\frac{3}{4} J_{r}-\mu\right) s_{i}^{\dagger} s_{i}+\left(\frac{1}{4} J_{r}-\mu+h\right) t_{+i}^{\dagger} t_{+i} \quad(\mathrm{~S} 10)\right. \\
& \left.+\left(\frac{1}{4} J_{r}-\mu\right) t_{0 i}^{\dagger} t_{0 i}+\left(\frac{1}{4} J_{r}-\mu-h\right) \bar{t}^{2}+\mu+\frac{1}{2} J_{l} \bar{t}^{4}\right] \\
& +\frac{1}{2} J_{l} \sum_{i}\left[\bar{t}^{2}\left(s_{i}^{\dagger} s_{i+1}+t_{0 i}^{\dagger} t_{0 i+1}+\text { H.c. }\right)-2 \bar{t}^{2} t_{-i}^{\dagger} t_{-i}\right]
\end{aligned}
$$

[S1] S. Ward, S. Furuya, D. Biner, K. W. Krämer, D. Cheptiakov, M. Boehm, D. F. McMorrow, T. Giamarchi, and Ch. Rüegg, unpublished.

[S2] I. Affleck, T. Kennedy, E. H. Lieb, and H. Tasaki, Phys. Rev. Lett. 59, 799 (1987); Commun. Math. Phys. 115, 477 (1988).

[S3] B. Thielemann, Ph. D. thesis, ETH Zurich (2009).

[S4] Available at http://www.mantidproject.org.

[S5] R. A. Ewings, A. Buts, M. D. Le, J. van Duijn, I. Bustinduy, and T. G. Perring, Nuclear Instruments and Methods in Physics Research Section A: Accelerators, Spec- 
trometers, Detectors and Associated Equipment 834, 132 (2016).

[S6] M. Reigrotzki, H. Tsunetsugu, and T. M. Rice, J. Phys.: Condens. Matter 6, 9235 (1994).

[S7] B. Normand and Ch. Rüegg, Phys. Rev. B 83, 054415 (2011).

[S8] Ch. Rüegg, K. Kiefer, B. Thielemann, D. F. McMorrow, V. Zapf, B. Normand, M. B. Zvonarev, P. Bouillot, C. Kollath, T. Giamarchi, S. Capponi, D. Poilblanc, D. Biner, and K. W. Krämer, Phys. Rev. Lett. 101, 247202 (2008).

[S9] G. Xu, C. Broholm, D. H. Reich, and M. A. Adams, Phys. Rev. Lett. 84, 4465 (2000).

[S10] B. Bauer, L. D. Carr, H. G. Evertz, A. Feiguin, J. Freire, S. Fuchs, L. Gamper, J. Gukelberger, E. Gull, S. Guertler, A. Hehn, R. Igarashi, S. V. Isakov, D. Koop, P. N. Ma, P. Mates, H. Matsuo, O. Parcollet, G. Pawlowski, J. D. Picon, L. Pollet, E. Santos, V. W. Scarola, U. Schollwöck, C. Silva, B. Surer, S. Todo, S. Trebst, M. Troyer, M. L. Wall, P. Werner, and S. Wessel, J. Stat. Mech. P05001 (2011).

[S11] G. Vidal, Phys. Rev. Lett. 93, 040502 (2004).

[S12] S. R. White and A. E. Feiguin, Phys. Rev. Lett. 93, 076401 (2004).

[S13] A. J. Daley, C. Kollath, U. Schollwöck, and G. Vidal,
J. Stat. Mech.: Theor. Exp. P04005 (2004).

[S14] U. Schollwöck, Ann. Phys. 326, 96 (2011).

[S15] P. Bouillot, C. Kollath, A. M. Läuchli, M. Zvonarev, B. Thielemann, Ch. Rüegg, E. Orignac, R. Citro, M. Klanjšek, C. Berthier, M. Horvatić, and T. Giamarchi, Phys. Rev. B 83, 054407 (2011).

[S16] P. Bouillot, Ph. D. thesis, University of Geneva (2011).

[S17] W. Zheng, C. J. Hamer, R. R. P. Singh, S. Trebst, and H. Monien, Phys. Rev. B 63, 144410 (2001).

[S18] C. Knetter, K. P. Schmidt, M. Grüninger, and G. S. Uhrig, Phys. Rev. Lett. 87, 167204 (2001).

[S19] C. Knetter and G. S. Uhrig, Eur. Phys. J. B 13, 209 (2000).

[S20] C. Knetter, K. P. Schmidt, and G. S. Uhrig, Eur. Phys. J. B 36, 525 (2004).

[S21] M. B. Stone, I. A. Zaliznyak, T. Hong, C. L. Broholm, and D. H. Reich, Nature 440, 187 (2006).

[S22] R. Coldea, D. A. Tennant, K. Habicht, P. Smeibidl, C. Wolters, and Z. Tylczynski, Phys. Rev. Lett. 88, 137203 (2002).

[S23] B. Thielemann, Ch. Rüegg, H. Rønnow, A. M. Läuchli, J. S. Caux, B. Normand, D. Biner, K. W. Krämer, H. U. Güdel, J. Stahn, K. Habicht, K. Kiefer, M. Boehm, D. F. McMorrow, and J. Mesot, Phys. Rev. Lett. 102, 107204 (2009). 